\title{
SINERGISMO ENTRE DADOS ÓPTICOS E DE RADAR NO ESTUDO GEOLÓGICO DA REGIÃO DE BEZERRA-CABECEIRAS, GOIÁS
}

\author{
JOSELISA M. CHAVES ${ }^{1,2}$, EDSON E. SANO ${ }^{3}$, EDI M. GUIMARÃES ${ }^{2}$, \\ ARDEMÍRIO B. SILVA ${ }^{1,4}$ \& PAULO R. MENESES ${ }^{2}$
}

\begin{abstract}
SYNERGY BETWEEN OPTICAL AND SAR DATA FOR GEOLOGICAL MAPPING IN THE BEZERRA-CABECEIRAS REGION, GOIÁS STATE. Optical images provide spectral information related to chemical composition of targets, whereas the synthetic aperture radar (SAR) data present information related to geometry and dielectric properties of objects. Because it can combine complementary spectral data, the synergism between different sensors tends to improve the potential of remote sensing for geological mapping and other types of mapping. In this study, the synergy of the following aircraft and satellite data was evaluated for geological mapping of the Bezerra-Cabeceiras region, Goiás State: a scanned GEMS-1 SAR image from Radambrasil Project (Xband); two C-band RADARSAT images, with different incident angles and look direction; a L-band JERS-1 SAR image; and an optical Landsat 5-TM dataset. The following digital image processing techniques were analyzed: principal components, decorrelation, linear contrast and IHS color composite. In this study we obtained a high quality IHS color composite for lithological and structural mapping and the reduction of land use interference in the relatively recent Landsat-5 TM data. Such quality was demonstrated through the analyses of comission and omission errors, in addition to the evaluation of accuracy.
\end{abstract}

Keywords: optical-SAR synergy; geological mapping; IHS color composite, RADAMBRASIL GEMS; RADARSAT STANDARD, JERS-1 SAR, LANDSAT TM.

\begin{abstract}
Resumo As imagens ópticas fornecem informações espectrais relacionadas à composição química dos alvos, enquanto as imagens de radar de abertura sintética (SAR - Synthetic Aperture Radar) apresentam informações relativas à geometria e propriedades elétricas dos alvos. Por combinar dados espectrais complementares, o sinergismo entre esses dois tipos de dados tende a melhorar o potencial do sensoriamento remoto para mapeamento geológico e outros tipos de mapeamentos. Nesse estudo, o sinergismo dos seguintes dados aerotransportados e orbitais foi avaliado para mapeamento geológico da região de Bezerra-Cabeceiras, GO: uma imagem de SAR, escaneada do GEMS-1/Radambrasil (banda X); duas imagens do RADARSAT (banda C), com ângulos de incidência e direções de visada distintos; uma imagem do JERS-1 (banda L); e uma imagem óptica do Landsat 5-TM, bandas 1, 2, 3, 4, 5 e 7. As seguintes técnicas de realce e integração de imagens digitais foram analisadas: componentes principais, decorrelação, contraste linear e composição colorida por IHS. Os principais resultados desse estudo foram a obtenção de uma composição colorida integrada por IHS com excelente qualidade visual para discriminação de unidades geológicas e realce de estruturas geológicas e a redução da interferência do uso da terra (agricultura e pecuária) nas imagens relativamente recentes do Landsat 5-TM. Essa qualidade pôde ser comprovada através da análise de erros de comissão e omissão, além da acurácia.
\end{abstract}

Palavras-chaves: sinergismo, mapeamento geológico, IHS, RADAMBRASIL GEMS, RADARSAT-1 STANDARD, JERS-1 SAR, LANDSAT TM.

INTRODUÇÃO Os sistemas ópticos fornecem informações sobre a superfície terrestre nas faixas do espectro eletromagnético correspondentes ao visível e ao infravermelho. O sensor óptico mais conhecido é o Thematic Mapper (TM), presente nos satélites mais recentes da série Landsat (Lauer et al. 1997, Mika 1997, Goward et al. 2001). Em geologia, diversos estudos de mapeamento geológico e prospecção mineral têm sido conduzidos com esse sensor (e.g., Fraser 1991, Souza Filho \& Drury 1998). Embora apresentem cobertura repetitiva e sinótica (passa num mesmo local a cada 16 dias e uma cena do Landsat corresponde a uma área no terreno equivalente a $185 \mathrm{~km} \times 185 \mathrm{~km}$ ), além de resolução espacial de $30 \mathrm{~m}$, adequada para um grande número de aplicações geológi- cas, os dados do Landsat apresentam algumas limitações que podem restringir o seu uso. Por exemplo, as larguras das bandas dos sensores TM e ETM ${ }^{+}$(Enhanced Thematic Mapper Plus) dos satélites Landsat 5 e 7 e as suas localizações no espectro não são favoráveis à discriminação entre certos minerais, tais como argilas e carbonatos (Meneses \& Ferreira Jr. 2001). Além disso, os dados do Landsat são fortemente influenciados pelas condições atmosféricas do local e da hora da passagem do satélite. A geometria de imageamento entre o sensor, o alvo e o Sol também é outro aspecto que não pode ser negligenciado, quando se interpreta uma imagem óptica.

Uma alternativa ao uso do sensoriamento remoto óptico são os

1 - Grupo de Pesquisa Geociências e Gestão de Recursos Naturais - Área de Geociências, Depto. Exatas, UEFS, Km 03, BR-116, 44031-460, Feira de Santana, Bahia. e-mail: joselisa@uefs.br

2 - Instituto de Geociências, Universidade de Brasília, 70910-900, Brasília, Distrito Federal, e-mail: rxedi@unb.br e pmeneses@unb.br

3 - Embrapa Cerrados, BR-020, Km 18, Cx. Postal 08223, 73301-970, Planaltina, Distrito Federal. e-mail: sano@cpac.embrapa.br

4 - Instituto de Geociências, Unicamp, Cx. Postal 6152, Campinas, São Paulo, e-mail: barros@ige.unicamp.br 
dados de radar de abertura sintética (SAR - synthetic aperture radar), adquiridos na faixa espectral de microondas (comprimentos de onda de 1mm a 1m) (Werle 1998, Raney 1998). Existem atualmente quatro satélites de radar de abertura sintética que operam numa órbita polar, destacando-se os satélites ERS-2 (europeu) e RADARSAT (canadense) que foram lançados em 1995. Pelo menos mais dois satélites, com capacidade de múltipla polarização, estão previstos para serem lançados num futuro próximo, destacando-se o RADARSAT-2 e o PALSAR (Phased Array type L-band Synthetic Aperture Radar, Japão).

Os sistemas de SAR têm as vantagens de penetração nas nuvens, menor influência dos efeitos atmosféricos, capacidade de operação durante o dia ou a noite e independência em relação às condições de iluminação solar (Elachi 1988, Henderson \& Lewis 1998, Lewis et al. 1998). A principal desvantagem é a falta de um melhor entendimento sobre os processos físicos que efetivamente ocorrem quando os sinais de radar incidem num alvo geológico, agrícola ou com vegetação natural. Parte dessa deficiência é decorrente da inexistência de sensores comerciais de radar (escaterômetros) com custo acessível, o que impossibilita a realização de campanhas de validação no campo ou mesmo com o uso de aeronave (Moran et al. 2002). Apesar dessa dificuldade, alguns autores têm obtido resultados promissores com dados de radar nos estudos de prospecção e mapeamento geológico e análise estrutural (e.g. Singhroy 1992, Paradella et al. 1998, 2000).

As incertezas inerentes aos dois tipos de sensores têm aumentando o interesse da comunidade científica no uso do sinergismo entre dados ópticos e de radar. Nesse caso, a principal meta é aproveitar os fatores positivos de cada sistema na elaboração de produtos com maior quantidade de informações, pois os dados por eles registrados apresentam naturezas distintas e complementares. Logo, o seu uso combinado pode ser altamente favorável, pois as imagens de SAR tendem a enfatizar a informação espacial (estruturas e texturas), enquanto as imagens ópticas realçam a informação espectral.

Nesse estudo, um conjunto de imagens do Landsat 5-TM foi combinado com diferentes dados de SAR na melhoria da discriminação de litotipos da região de Bezerra-Cabeceiras (GO). Essa área é representada por três unidades sedimentares meso a neoproterozóicas dos Grupos Paranoá e Bambuí e Formação Jequitaí, sendo importante para o entendimento da geologia da Faixa Brasília.

ABORDAGEMMETODOLÓGICA Área de estudo Aáreade estudo localiza-se entre a vila de Bezerra (município de Formosa, $\mathrm{GO}$ ) e a cidade de Cabeceiras (GO), entre as coordenadas $15^{\circ} 29^{\prime} 05^{\prime \prime}$ e $15^{\circ} 40^{\prime} 29^{\prime \prime}$ de latitude sul e $47^{\circ} 00^{\prime} 26^{\prime \prime}$ e $47^{\circ} 08^{\prime} 44^{\prime \prime}$ de longitude oeste (Fig. 1). Do ponto de vista geológico, a área-teste situa-se a oeste do cráton São Francisco e a leste do maciço de Goiás, na zona externa da faixa Brasília. As unidades aflorantes mais antigas são os quartzitos, ritmitos e arcóseos e, subordinadamente, carbonatos pertencentes ao Grupo Paranoá (Guimarães 1997). Essas unidades são recobertas, em discordância erosiva, por rochas da Formação Jequitaí, composta tanto por níveis delgados e descontínuos de diamictitos, como por ritmitos, margas e arenitos. Sobre essa formação ou sobre as rochas do Grupo Paranoá, repousam as rochas pelíticas e carbonáticas do Grupo Bambuí, onde os termos psamíticos encerram a seqüência.

Dados de sensoriamento remoto: aquisição e processamento As seguintes imagens de satélite e de aeronave (Fig. 2) foram analisa- das nesse estudo: uma cena do Landsat 5-TM, órbita 221, ponto 71, obtida em 30 de maio de 1997, bandas 1, 2, 3, 4, 5 e 7; duas imagens do satélite RADARSAT, órbitas ascendente e descendente, modo standard de imageamento e ângulos de incidência de $24-31^{\circ}(\mathrm{S} 2)$ e $45-49^{\circ}(\mathrm{S} 7)$, obtidas em 13 de janeiro de 1999 e 23 de maio de 2000, respectivamente; uma imagem de radar do satélite JERS-1, de 10 de março de 1994 e uma imagem aerotransportada, folha Brasília (SD.23), obtida pela plataforma GEMS-1, utilizada pelo Projeto Radambrasil (Tabela 1).

O JERS-1 foi lançado pela NASDA (National Space Development Agency of Japan) em 11 de fevereiro de 1992 e seu sistema SAR deixou de adquirir dados no final de 1998. O JERS-1 SAR operava na banda $\mathrm{L}$ (comprimento de onda $=23,5 \mathrm{~cm}$ ), polarização $\mathrm{HH} \mathrm{e}$ ângulo de incidência entre $36^{\circ}$ (alcance próximo) e $41^{\circ}$ (alcance distante). A faixa de imageamento era de $75 \mathrm{~km}$, com uma resolução espacial nominal de $18 \mathrm{~m}$.

O RADARSAT-1 foi lançado pela Canadian Space Agency (CSA) em 5 de novembro de 1995. Possui uma órbita heliossíncrona e quase polar e opera em banda $\mathrm{C}(5,6 \mathrm{~cm})$, polarização $\mathrm{HH}$, a uma altitude de $798 \mathrm{~km}$. Os ângulos de incidência variam de $10 \mathrm{a} 60^{\circ}$, as faixas de imageamento de 45 a $500 \mathrm{~km}$ e a resolução espacial de 8 a $100 \mathrm{~m}$ (modos fine $=8 \mathrm{~m}$; standard $=25 \mathrm{~m}$; wide $=30 \mathrm{~m} ; \mathrm{e}$ ScanSAR $=50$ ou $100 \mathrm{~m}$ ). A empresa privada denominada RADARSAT International (RSI) é a responsável pelo processamento, comercialização e distribuição desses dados.

O GEMS-1 foi o sistema de radar aerotransportado usado no Projeto Radambrasil, com banda X $(3,1 \mathrm{~cm})$ e polarização HH. Possui ângulo de incidência de $45-77^{\circ}$ e resolução espacial de 16 metros. No Projeto Radambrasil, as imagens foram analisadas no formato analógico para mapeamentos de geologia, geomorfologia, pedologia e de vegetação. No final da década de 90 , essas imagens foram escanerizadas, possibilitando o seu uso no formato digital.

Os efeitos atmosféricos na imagem do Landsat 5-TM foram minimizados através do método da subtração pelo pixel mais escuro, conhecido como método do mínimo histograma (Kaufman e Sendra 1988; Teillet e Fedosejevs 1995). A imagem foi ainda georreferenciada para o sistema de coordenadas UTM (Universal Transversa de Mercator), datum SAD-69 (South American Datum 1969) utilizando-se 100 pontos de controle obtidos de cartas topográficas 1:100.000 da área de estudo.

Com relação às imagens do RADARSAT, os dados de atitude, efemérides, geometria de visada e um modelo numérico de terreno gerado a partir das curvas de nível da área de estudo, extraídas das cartas planialtimétricas na escala 1:100.000, foram utilizados para ortorretificá-las. Para isso, foi utilizado o pacote de programas denominado "Ortho \& DEM", disponível no software comercial PCI (1999, versão 6.3). Para as imagens do JERS-1 e GEMS-1, foi realizada apenas o georreferenciamento simples, isto é, sem ortorretificação, pois não havia disponibilidade das informações de atitude, efemeridade e geometria de visada, necessárias para esse procedimento.

Diferentes filtros (Média, Mediana, Frost, Lee, etc) e tamanhos variados de máscaras $(3 \times 3,5 \times 5,7 \times 7$ pixels $)$ foram testados para atenuar o speckle, um tipo de ruído que é inerente aos dados orbitais de radar. As imagens de SAR e Landsat/TM, a princípio, não são compatíveis tanto em relação ao número de bits, representativos dos níveis de cinza de cada pixel, quanto na resolução espacial do pixel no terreno (Tabela 1). As imagens de SAR são obtidas de uma visada lateral diferente da imagem óptica, que é cônica em visada nadir. Em função dessas diferenças, é natural 

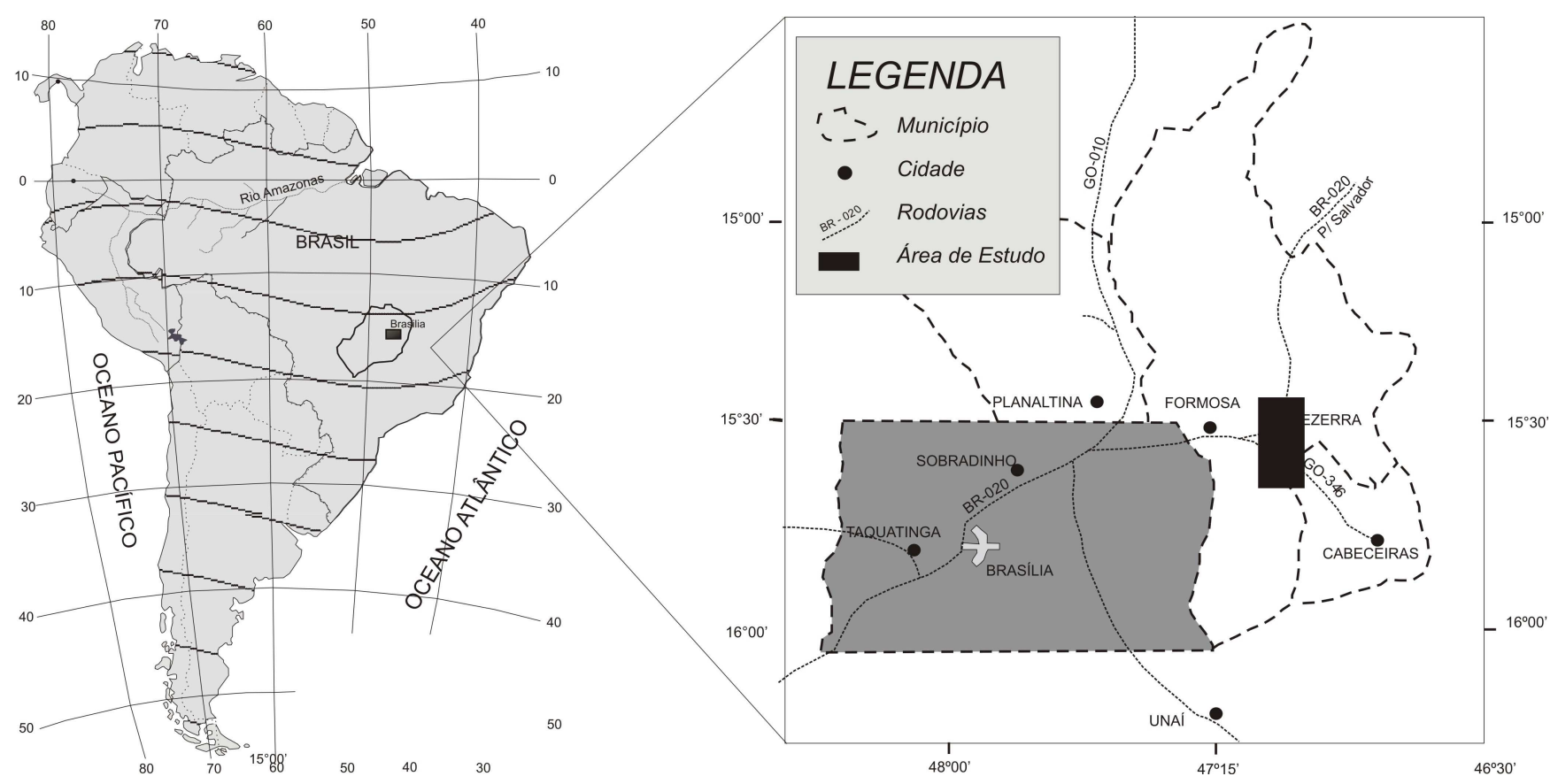

Figura 1 - Mapa de localização da área de estudo.

Tabela 1 - Características das imagens aerotransportadas e de satélite, adquiridas sobre a região de Bezerra-Cabeceiras, GO.

\begin{tabular}{|c|c|c|c|c|c|c|}
\hline \multirow{2}{*}{ Plataforma } & Sensor & $\begin{array}{c}\text { Data de } \\
\text { Aquisição }\end{array}$ & $\begin{array}{c}\text { Angulo de } \\
\text { Incidência }\end{array}$ & $\begin{array}{c}\text { Angulo de } \\
\text { Visada }\end{array}$ & $\begin{array}{c}\text { Resolução } \\
\text { Espacial } \\
\text { Nominal }\end{array}$ & $\begin{array}{c}\text { Bandas } \\
\text { Espectrais }\end{array}$ \\
\hline Landsat & TM & $30 / 05 / 97$ & Nadir & ---- & $30 \mathrm{~m}$ & $1,2,3,4,5 \mathrm{e} 7$ \\
\hline Radarsat & Standard S7 & $23 / 05 / 00$ & $45-49^{\circ}$ & $78^{\circ}$ & $25 \mathrm{~m}$ & $\mathrm{C}(5,6 \mathrm{~cm})$ \\
\cline { 2 - 7 } & Standard S2 & $13 / 01 / 99$ & $24-31^{\circ}$ & $282^{\circ}$ & $25 \mathrm{~m}$ & $\mathrm{C}(5,6 \mathrm{~cm})$ \\
\hline JERS-1 & SAR & $10 / 03 / 94$ & $36-41^{\circ}$ & $282^{\circ}$ & $18 \mathrm{~m}$ & $\mathrm{~L}(23,5 \mathrm{~cm})$ \\
\hline GEMS-1 & SAR & $1975 / 1976$ & $45-77^{\circ}$ & ---- & $16 \mathrm{~m}$ & $\mathrm{X}(3,1 \mathrm{~cm})$ \\
\hline
\end{tabular}

que existam distorções entre as imagens, sendo necessária à realização de alguns pré-processamentos e reamostragem dos pixels, para uma compatibilização e futura integração desses dados. Para o processamento das imagens, foram utilizados os programas ENVI (Versão 3.2) e PCI (Versão 6.3). Dessa forma, as imagens orbital e aerotransportada de SAR foram escalonadas de 16 para 8 bits, através de equalização linear, e reamostradas para um espaçamento de pixel de 30 metros, através do uso de um interpolador bilinear. Durante as correções geométricas foram observados os valores de acurácia (rms) do georreferenciamento.

A combinação dos dados do Landsat com as imagens do radar foi realizada através da transformação no espaço IHS de cores (Harris e Murray 1990). Essa transformação associa as bandas espectrais com as três cores primárias (vermelho, verde e azul), num processo aditivo onde as cores são relacionadas aos valores triestímulos dos monitores através das intensidades de energia geradas no tubo de imagem (Meneses et al. 1995). A transformação das cores de um sistema RBG para um IHS segue modelos representados geometricamente por cones (ou hexacones) e cilindros. Nessa pesquisa o modelo que apresentou melhores resultados foi o modelo de cones, que segundo Paradella et al. (2001) são os modelos que tem apresentado mais adequação, uma vez que a percepção de cores decresce com a intensidade, ou seja, a influên- cia do matiz e da saturação são insignificantes sob valores baixos de intensidade.

O procedimento de transformação RGB para IHS envolve três etapas de trabalho. A primeira compreende a transformação dos componentes R (red, vermelho), G (green, verde) e B (blue-azul) para os componentes I (intensity - intensidade, representa o brilho da imagem), $\mathrm{H}$ (hue - matiz, representa a cor propriamente dita) e S (saturation - saturação, representa a pureza da cor) a partir de três bandas do sistema óptico. As seguintes combinações de dados ópticos foram testadas nessa etapa: três bandas originais; três bandas decorrelacionadas; e combinação de bandas por componentes principais parcial e total de bandas.

A segunda etapa do sinergismo envolve a substituição dos componentes I, H e/ou S por uma imagem de radar (original ou processada) ou uma imagem componente do Landsat. Nesse estudo, foram testadas as imagens componentes, mais especificamente, a primeira componente principal obtida incluindo-se todas as imagens de SAR (CP1 SAR) e a CP1 obtida incluindo-se as seis bandas do Landsat 5-TM (CP1 TM). Finalmente, a última etapa do sinergismo é a transformação inversa IHS $\rightarrow$ RGB, realizada sem interação usuário-máquina. Com isso, obtém-se uma composição colorida que mantém a informação espectral do sistema óptico e a informação espacial da imagem de radar.

A seleção das três melhores bandas originais do Landsat 5-TM para serem incluídas no sinergismo foi realizada através do cálculo do OIF (Optimum Index Factor). Essa técnica, desenvolvida por Chavez et al. (1982), permite maximizar a quantidade de informações contidas na composição colorida e minimizar as informações perdidas. Além das cenas originais, bandas do TM, realçadas por componentes principais e por decorrelação, também foram incluídas na combinação Landsat-SAR.

A técnica de transformação estatística por componentes principais envolve uma rotação e translação de eixos num hipotético espaço de atributos de um sistema de coordenadas da função densidade de probabilidade, produzindo novos eixos ou componentes ortogonais ou independentes (Gillespie 1980; Meneses et 

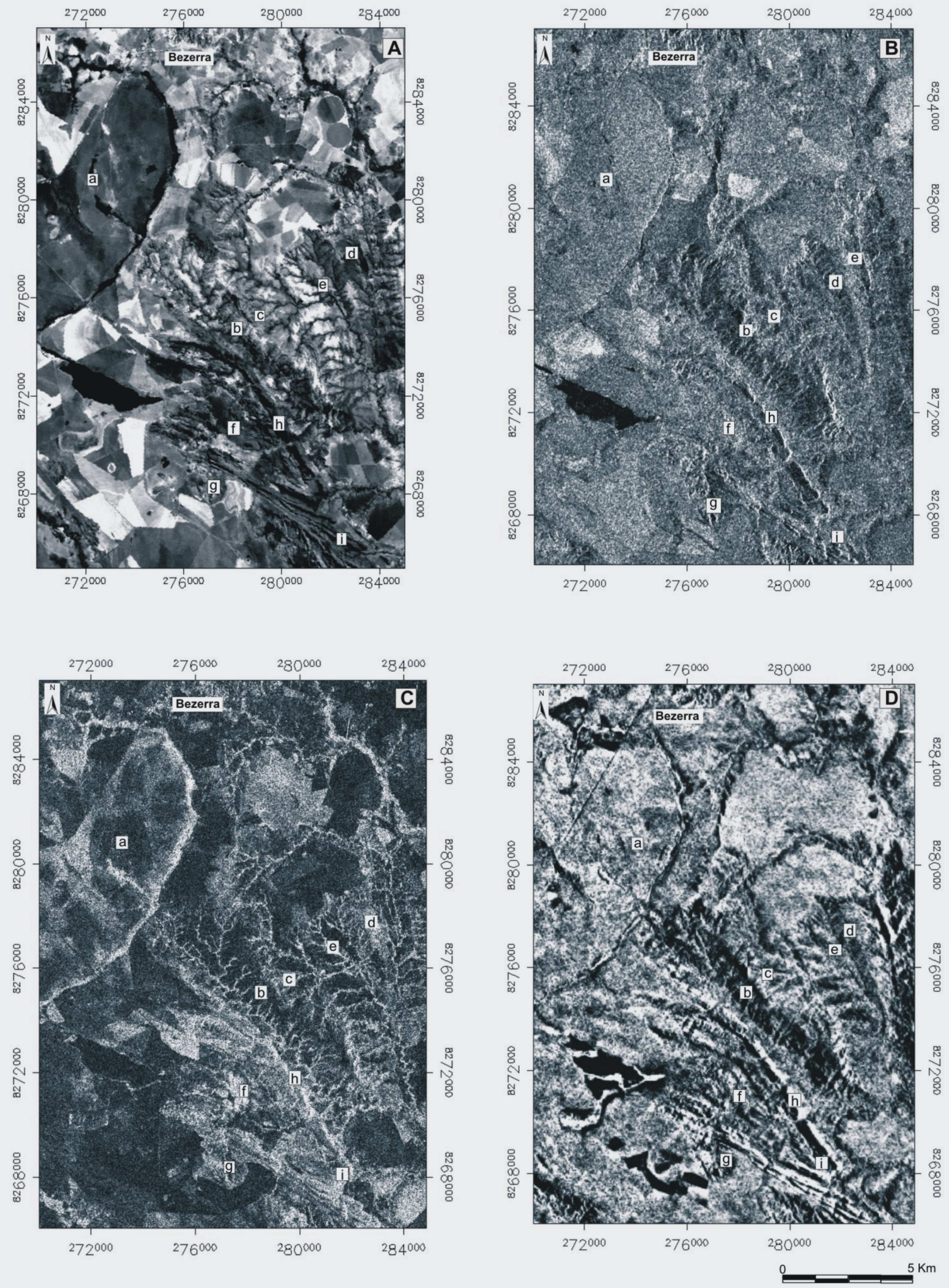

Figura 2 - Imagens orbitais e aerotransportadas analisadas no estudo de sinergismo.A - Landsat 5-TM, banda 5; B - Radarsat, modo de imageamento S2; C - JERS-1 SAR; D - GEMS-1 SAR. As letras representam: a - Cobertura Cenozóica; $b$ - Formação Três Marias escarpa; c - Formação Três Marias superficie; d-Formação Serra da Saudade escarpa; e - Formação Serra da Saudade superficie; $f$ - Formação Sete Lagoas; $g$ - Ritmito Superior; $h$ - nível arcoseano e $i$ - estruturas geológicas (Sinclinais de Raizama). 
al. 1995). Essa técnica reduz a dimensionalidade dos dados e os novos componentes gerados, conhecidos como componentes principais, apresentam quantidades decrescentes de informações. A análise por componentes principais parciais tem como base a Selective Principal Component Analysis proposta por Chavez Jr. et al. (1984) e Chavez Jr. e Kwarting (1994). Considerando-se que a análise visual da imagem óptica mostrou uma nítida correlação com vegetação e unidades geológicas, optou-se por testar, na análise de componentes principais, os seguintes subconjuntos de bandas: TM 1,23 (Grupo 1) e TM 5, 7 (Grupo 2). As primeiras componentes principais desses grupos foram associadas com a banda 4 na transformação IHS. Dessa forma buscou-se modelar o espectro da vegetação.

A técnica de decorrelação (Gillespie et al. 1986) reduz a correlação entre bandas através de uma rotação dos eixos das imagens e de uma equalização inversa dos eixos. As imagens decorrelacionadas normalmente conservam as informações espectrais originais, o que não ocorre na transformação por componentes principais.

Estratégia de análise do sinergismo Em função da inexistência de um mapa geológico semidetalhado da área de estudo, decidiuse pela confecção do mesmo, através de reconhecimento geológico em campo, do uso de um esboço geológico apresentado por Guimarães (1997) e da interpretação visual de uma composição colorida de três bandas originais R4, G5 e B7 do TM.

Em seguida, as interpretações geológicas derivadas dos cinco diferentes produtos integrados entre Landsat e SAR foram comparadas quantitativamente com o referido mapa geológico, através dos cálculos de erros de comissão (EC) e de omissão (EO), além da acurácia total (AT) (Silva 1999). EC refere-se à associação de pixels a classes temáticas que não existem no terreno, enquanto EO é a não associação de pixels a classes temáticas efetivamente existentes no terreno. A acurácia total foi analisada levando-se em conta as diferentes possibilidades de fontes de erros (EC e OC). Os cálculos de EC, EO e AT, obtidos pelo pacote de programa Idrisi, versão 3.2 e pela planilha Excel, envolveram 11 categorias representativas dos litotipos da região de estudo.

RESULTADOS E DISCUSSÃO Pré-processamento de imagens Com relação à etapa de pré-processamento de imagens, vale ressaltar que o erro de georreferenciamento da cena do Landsat 5-TM ficou em torno de $5,7 \mathrm{~m}$, bem menor que a resolução espacial nominal do TM $(30 \mathrm{~m})$.

As imagens RADARSAT foram ortocorrigidas seguindo as etapas definidas por Pietsch (1993). Nesse caso, ao invés de corrigir, no local, a imagem ajustando um modelo polinomial que leva em conta os pontos de controle, o modelo geométrico corrige globalmente a imagem, considerando as distorções da plataforma, do sensor, da Terra e da projeção cartográfica. Para essa correção geométrica, foram feitas coletas de pontos de controle, com coordenadas X, Y e Z. Para a imagem RADARSAT S2, foram obtidos 25 pontos, cujo modelo matemático gerou um Erro Quadrático Médio(RMS) de 7,64m (0,61 pixel). Já para a imagem RADARSAT S7, foram coletados 20 pontos, e o modelo matemático gerou um RMS em torno de 8,66m ( 0,69 pixel). Embora os modelos matemáticos apresentassem valores de erros quadráticos baixos, utilizouse, na ortocorreção, um tamanho de pixel equivalente a $30 \mathrm{~m}$, buscando manter a integridade geométrica e radiométrica para a fusão desses dados com as imagens Landsat/TM. O sistema adotado na correção geométrica foi a projeção Universal Transversa de
Mercator(UTM), com datum South American 1969 (SAD-69). No processo de ortocorreção para a remoção dos erros devido às mudanças de elevação, foi levado em conta o Modelo Digital do Terreno gerado no programa Geosoft e importado para o banco de dados do PCI. Para a reamostragem final da imagem, pode-se selecionar um filtro de eliminação do speckle substituindo os interpoladores (vizinho mais próximo, bilinear ou bicúbico), produzindo uma imagem ortocorrigida e, com efeito, de speckle minimizado, evitando múltiplas interpolações (Amaral \& Shimabukuro 1997).

Na correção geométrica da imagem JERS-1, levou-se em conta à mesma projeção usada para as demais imagens, ou seja, UTM, com datum SAD-69. Foram coletados 25 pontos, obtendo-se um RMS de aproximadamente $6 \mathrm{~m}$. Já para a imagem SAR do GEMS-1, o pré-processamento consistiu na correção geométrica da coleta de pontos de controle extraídos do programa PCI (1999), tendo como base cartográfica, as folhas topográficas de Santa Rosa (SD.23-Y-C-II), Brasília (SD.23-Y-C-IV), Formosa (SD.23-Y-C-V), Cabeceiras (SD.23-Y-C-VI) e dados vetoriais de estradas e drenagens. Foram coletados 25 pontos, com acurácia do RMS abaixo de 16 metros, para o modelo polinomial de primeira ordem. Esse valor foi selecionado como tamanho do pixel, tendo sido re-amostrado para 30 metros na integração das imagens.

Quanto à redução do speckle nas imagens orbitais de SAR, o filtro Frost (Frost et al. 1982), com máscara de 3 pixels $\mathrm{x} 3$ pixels, foi o que apresentou o melhor desempenho.

Mapa geológico semidetalhado da área de estudo Com base em 81 pontos de levantamento de campo e análise da composição colorida Landsat 5-TM, foi confeccionado o mapa geológico semidetalhado (escala de 1:60.000, Fig. 3). As unidades litoestratigráficas encontradas na área de estudo correspondem às rochas detríticas e carbonáticas dos Grupos Paranoá e Bambuí, além da Formação Jequitaí e coberturas cenozóicas.

O Grupo Paranoá é constituído por um empilhamento de quartzitos e pelitos, com participação menor de arcóseos, quartzitos glauconíticos e carbonatos. As unidades desse grupo, mapeadas por Guimarães (1997), da base para o topo, foram: Nível Arcoseano, formado por arcóseos arroxeados, de granulação média; e Ritmito Superior, caracterizado pela presença de quartzitos glauconíticos, além de incluir rochas dolomíticas com estromatólitos do tipo Conophyton.

A unidade basal do Grupo Bambuí é constituída pela Formação Sete Lagoas que apresenta ampla variação de espessura (60m a $100 \mathrm{~m})$ e de fácies, sendo constituída por folhelhos, margas e rochas carbonáticas. Sobreposta a essa formação, ocorre em contato brusco, a Formação Serra de Santa Helena, com espessura menor que 80 me constituída por siltitos e arenitos de granulação fina, ambos subquartzosos formando cristas de relevo alongadas.

A Formação Serra de Santa Helena é recoberta pela Formação Lagoa do Jacaré a qual tem espessura em torno de $50 \mathrm{~m}$ e é constituída por folhelhos e calcários, em geral, pretos, argilosos, e com níveis oolíticos. As áreas de afloramento são pouco extensas e seu contato superior com a Formação Serra da Saudade é bem marcado. Essa formação é constituída, predominantemente, por material pelítico, com pequenas lentes carbonáticas, formando um ritmito fino, alternando-se com lâminas sílticas subquartzosas e argilosas cinza-escuras e esverdeadas. No topo, passam a predominar os siltitos cada vez mais grossos, até o aparecimento das primeiras camadas arenosas que marcam o contato com a unidade superior do Grupo Bambuí (Formação Três Marias). 
A Formação Três Marias tem espessura de quase 200 metros, apresenta feições características, como a alta resistência física e química e alteração esferoidal por intemperismo. É constituída por intercalações de siltitos e arenitos subquartzosos de granulação fina a média, de coloração cinza-escura, em geral esverdeada, com níveis arroxeados.

As estruturas sedimentares e as relações de fácies definidas por Guimarães (1997) possibilitaram inferir que os sedimentos do Grupo Paranoá acumularam-se em área tectonicamente estável, circundada por áreas de relevo relativamente baixo, em condições de inter a intramaré. Os sedimentos do Grupo Bambuí foram depositados predominantemente sobre plataforma, em uma bacia com taxas de subsidência e de sedimentação relativamente altas, limitada, em parte, por relevo, também, relativamente alto. Quanto à rocha-fonte, Guimarães (1977) sugeriu uma fonte cratônica para o Grupo Paranoá. O Grupo Bambuí, por apresentar sedimentos terrígenos bastante imaturos, é originário de áreas-fonte formadas por rochas metassedimentares, ígneas (ou metaígneas) de composição intermediária a básica. A composição química de rocha total indicou sedimentação sobre a margem continental passiva para o Grupo Paranoá e para as rochas do Grupo Bambuí, uma composição compatível com a margem continental ativa.

Na região de Bezerra-Cabeceiras, entre os Grupos Paranoá e Bambuí, ocorre a Formação Jequitaí, com espessura inferior a 50 metros, por vezes inferior a um metro, caracterizada pelos diamictitos, contendo também ritmitos e rochas dolomíticas. Essa formação é recoberta por folhelhos e rochas carbonáticas da unidade basal do Grupo Bambuí.

As imagens de SAR realçaram as unidades de carbonatos e pelitos, em função, principalmente, dos aspectos texturais, impressos pela distribuição das drenagens e da geomorfologia, além da unidade de cobertura, marcada pela utilização do solo. As principais estruturas observadas nas imagens são estratificação $\left(\mathrm{S}_{0}\right)$, um sistema de falhas e fraturas bem regular e dobra sinforme (Figs. 3 e 4). No contato entre as formações foram inferidas falhas de empurrão, observadas pela variação litológica, expresso pelos padrões fotogeológicos distintos. A dobra do tipo sinforme foi identificada na porção SW, onde aflora a base do Grupo Bambuí com o Grupo Paranoá, possui eixo com direção NW-SE. Fraturas e falhas, com traços retilíneos são observadas, principalmente na parte sudeste da área, com direções preferenciais E-W.

Cálculo de OIF Os cálculos de OIF (Tabela 2) indicaram que o conjunto das bandas 3, 4 e 5 do Landsat 5-TM, seguida pela combinação 4, 5 e 7, seriam as mais favoráveis para o mapeamento geológico da área de estudo. Analisando-se visualmente essas duas combinações, optou-se por utilizar o conjunto 4,5 e 7 , que permite a separação entre as unidades com carbonatos e as com cobertura detrítica. Essa combinação permite ainda a separação das seguintes unidades litológicas: unidades carbonáticas - as Formações Sete Lagoas, Lagoa do Jacaré, Nível Arcóseo e Jequitaí; unidades pelíticas - Formações Três Marias, Serra da Saudade, Serra de Santa Helena e Ritmito Superior; e a unidade de cobertura cenozóica, que ocorre em áreas planas (unidade geomorfológica definida pelo compartimento I por Chaves 2002) e associada a uma intensa atividade agrícola. A interpretação visual da composição colorida das bandas 4, 5 e 7 do TM permitiu ainda a subdivisão das Formações Três Marias e Serra da Saudade em dois tipos distintos de paisagem: em zonas de escarpa e em áreas planas, paralelas ao acamamento regional.

Portanto, os conjuntos das bandas 4, 5 e 7, originais e decorrelacionadas, foram selecionadas para gerar a transformação RGB $\rightarrow$ IHS, já no processo de sinergismo entre Landsat 5TM e SAR (Tabela 3). Os outros conjuntos adicionais de bandas que foram incluídos nessa referida transformação foram: CP1 das bandas 1, 2 e 3 (visível) do Landsat 5-TM (com e sem decorrelação); CP1 das bandas 5 e 7 (infravermelho médio) do Landsat 5-TM (com e sem decorrelação); e a banda 4 (infravermelho próximo). Dessa forma, buscou-se modelar o espectro da vegetação, contemplando as faixas espectrais correspondentes à absorção por pigmentos fotosssintéticos (bandas 1, 2 e 3), absorção por água, celulose e compostos com carbono (bandas 5 e 7 ) e espalhamentos devido às interfaces água-ar e paredes das células (banda 4).

$\mathrm{Na}$ Tabela 3, podem ser observadas as diferentes substituições no processo de integração pela transformação via IHS. Como bandas iniciais no espaço RGB, foram colocadas quatro opções: TM 754 (original e decorrelacionada) e CP1 (TM1,2,3), TM 4, CP 1 (TM5,7) (original e decorrelacionada). Essas combinações de bandas foram escolhidas por apresentarem os melhores resultados visuais. Em seguida foram testadas quatro opções de substituição de componentes IHS, para cada uma das quatro combinações de bandas testadas, antes da transformação inversa para o espaço RGB. Dois conjuntos de imagens SAR foram testados, através da técnica de componentes principais: todas as imagens de SAR (RADARSAT, modos S2 e S7; JERS-1; e GEMS-1); e uma imagem para cada frequência (GEMS-1, banda X; RADARSAT S7, banda C; e JERS-1, banda L).

Na Opção 1, a componente I foi substituída pela componente CP1 SAR e as componentes S e H mantidas. Com essa operação, houve uma melhora na discriminação das lineações de drenagem e de relevo. As estruturas e as unidades associadas com relevo acentuado (nível Arcoseano; Formações Três Marias e Serra da Saudade, escarpa; Formação Sete Lagoas; Ritmito Superior) foram mais bem definidas. No entanto, houve uma redução acentuada no contraste espectral.

Na Opção 2, optou-se por substituir a componente I pela componente CP1 SAR. A banda $\mathrm{H}$ não foi substituída, enquanto na componente $\mathrm{S}$ foi colocada uma imagem sintética com nível de cinza constante. Com isso, obteve-se uma imagem com maior nitidez para a definição de estruturas, embora, em algumas partes, apresenta-se uma interferência acentuada do uso do solo, oriunda da imagem TM. As Opções 3 e 4 (Tabela 3) mostraram-se mais indicadas para análises de vegetação e solo

A Opção 1 foi a que apresentou a melhor discriminação litológica e estrutural. Baseado nessa opção, confeccionou-se um novo mapa geológico (Fig. 4). A comparação dos dois mapas geológicos elaborados nesse estudo (Figs. 3 e 4) permitiu o cálculo dos valores de EC, EO e AT, os quais são apresentados na forma de validação cruzada (Tabela 4).

Os erros calculados de comissão podem ser qualitativamente agrupados em menores que 0,22 (erro reduzido), de 0,23 a 0,40 (erro razoável) e maiores que 0,41 (erro acentuado). Baseado nesse critério, pode-se concluir que as unidades mapeadas como Lagoa do Jacaré, Jequitaí, cobertura cenozóica e Sete Lagoas possuem mais de $80 \%$ de chance de serem confirmadas no mapa geológico. Isso significa que os critérios utilizados nos processamentos digitais de imagens e as interpretações deles derivados, podem ser classificados como sendo excelentes.

As unidades Três Marias Superior, Serra de Santa Helena, Serra da Saudade escarpa e Ritmito superior possuem de 60 a $75 \%$ de chance de serem confirmadas no mapa geológico. Tais valores podem ser considerados razoáveis, no entanto, processamentos 


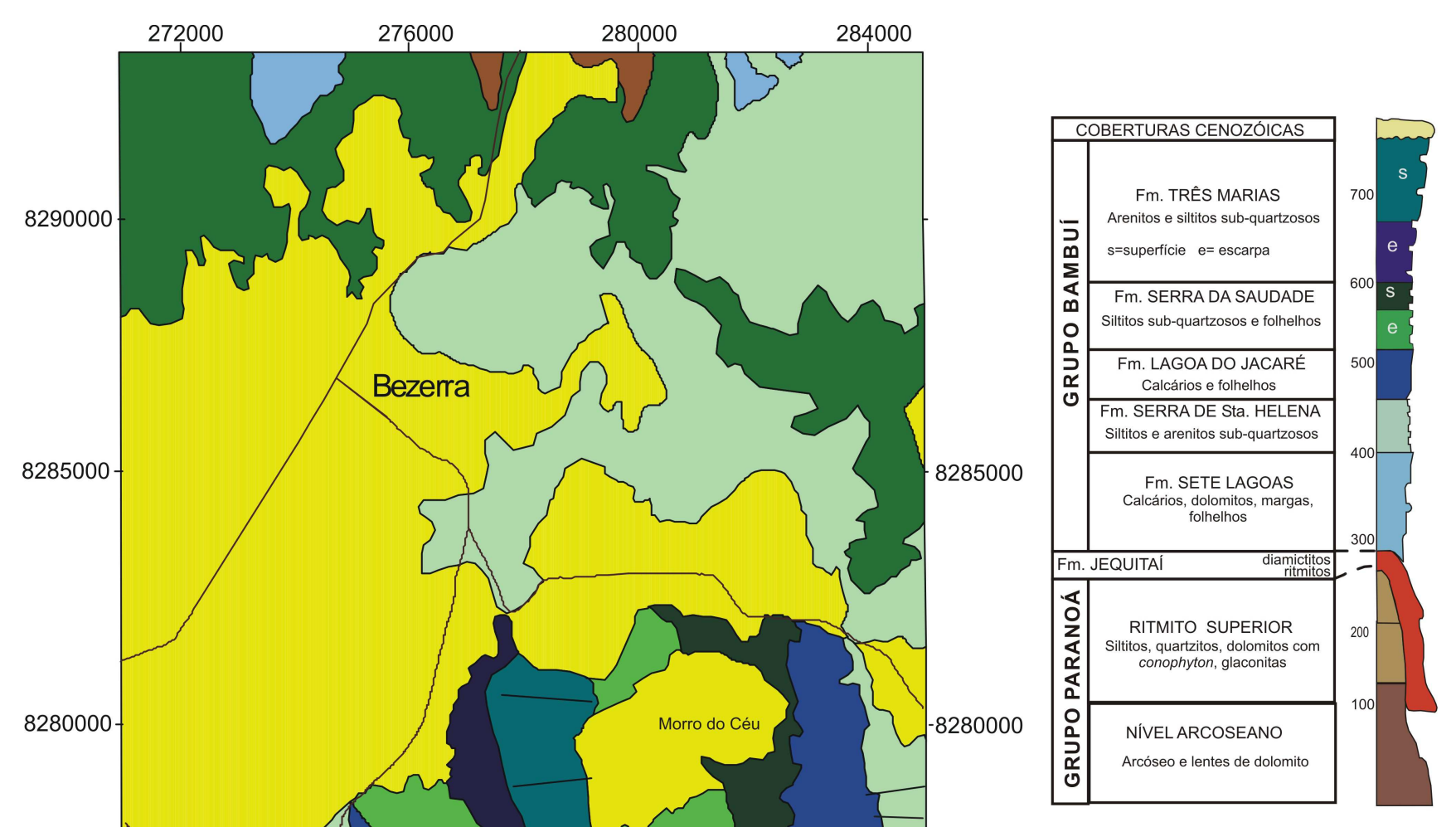

\section{LEGENDA}

8275000

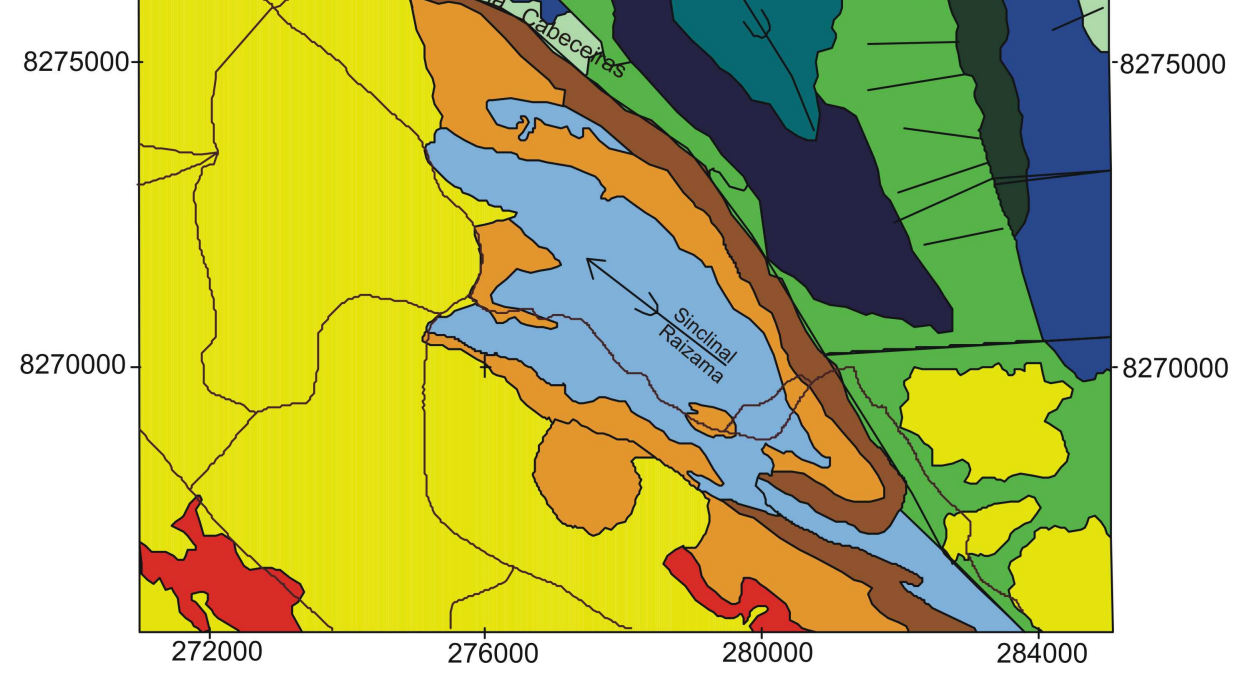

Figura 3 - Mapa geológico da região de Bezerra-Cabeceiras (GO). Adaptado e ampliado de Guimarães (1997).

digitais e interpretações respectivamente mais elaboradas e mais acuradas podem ser implementadas e obtidos menores erros. As unidades Nível Arcoseano e Três Marias escarpa apresentaram um elevado erro de comissão, indicando que existe uma chance menor que 55\% dessas unidades serem confirmadas no mapa geológico. Provavelmente este objetivo não seria atingido, mesmo utilizando-se de processamentos digitais mais sofisticados que melhorem a capacidade de interpretação.

A acurácia total, que reflete o exame global dos erros de omissão e, comissão, foi de 0.77 , indicando que as técnicas utilizadas e a interpretação dos produtos derivados dos processamentos digitais foram muito boas.

CONSIDERAÇÕES FINAIS Os resultados da integração da imagem do Landsat 5-TM com as imagens de SAR, com três bandas diferentes (X, $\mathrm{C} \mathrm{e} \mathrm{L}$ ), mostrou um bom potencial na discriminação de unidades litológicas e estruturais da região de BezerraCabeceiras (GO). A análise visual dos produtos integrados mostrou que as imagens de SAR são importantes no realce de feições topográficas e da rede de drenagem, contribuindo de forma ex- 
Tabela 2 - Valores de OIF (Optimum Index Factor) calculados para as combinações de três bandas espectrais do Landsat 5-TM da região de Bezerra-Cabeceiras, GO.

\begin{tabular}{|c|c|c|c|c|c|c|c|}
\hline $\begin{array}{c}\text { Composições } \\
\text { Coloridas }\end{array}$ & OIF & Rank & $\begin{array}{c}\text { Rank } \\
\text { visual }\end{array}$ & $\begin{array}{c}\text { Composições } \\
\text { Coloridas }\end{array}$ & OIF & Rank & $\begin{array}{c}\text { Rank } \\
\text { visual }\end{array}$ \\
\hline 345 & 30,5 & 1 & 1 & 157 & 13,7 & 11 & - \\
\hline 457 & 29,9 & 2 & 2 & 135 & 13,6 & 12 & - \\
\hline 145 & 26,4 & 3 & - & 257 & 13,6 & 13 & - \\
\hline 347 & 24,4 & 4 & 6 & 235 & 13,4 & 14 & - \\
\hline 245 & 23,7 & 5 & - & 124 & 12,6 & 15 & - \\
\hline 147 & 20,4 & 6 & 3 & 125 & 11,7 & 16 & - \\
\hline 134 & 18,7 & 7 & 5 & 137 & 9,0 & 17 & - \\
\hline 247 & 18,4 & 8 & - & 237 & 8,6 & 18 & - \\
\hline 234 & 16,2 & 9 & 4 & 127 & 7,2 & 19 & - \\
\hline 357 & 14,7 & 10 & - & 123 & 6,5 & 20 & - \\
\hline
\end{tabular}

Tabela 3 - Opções de substituição do componente intensidade (I) no processo de sinergismo entre as imagens do Landsat 5-TM e de SAR.

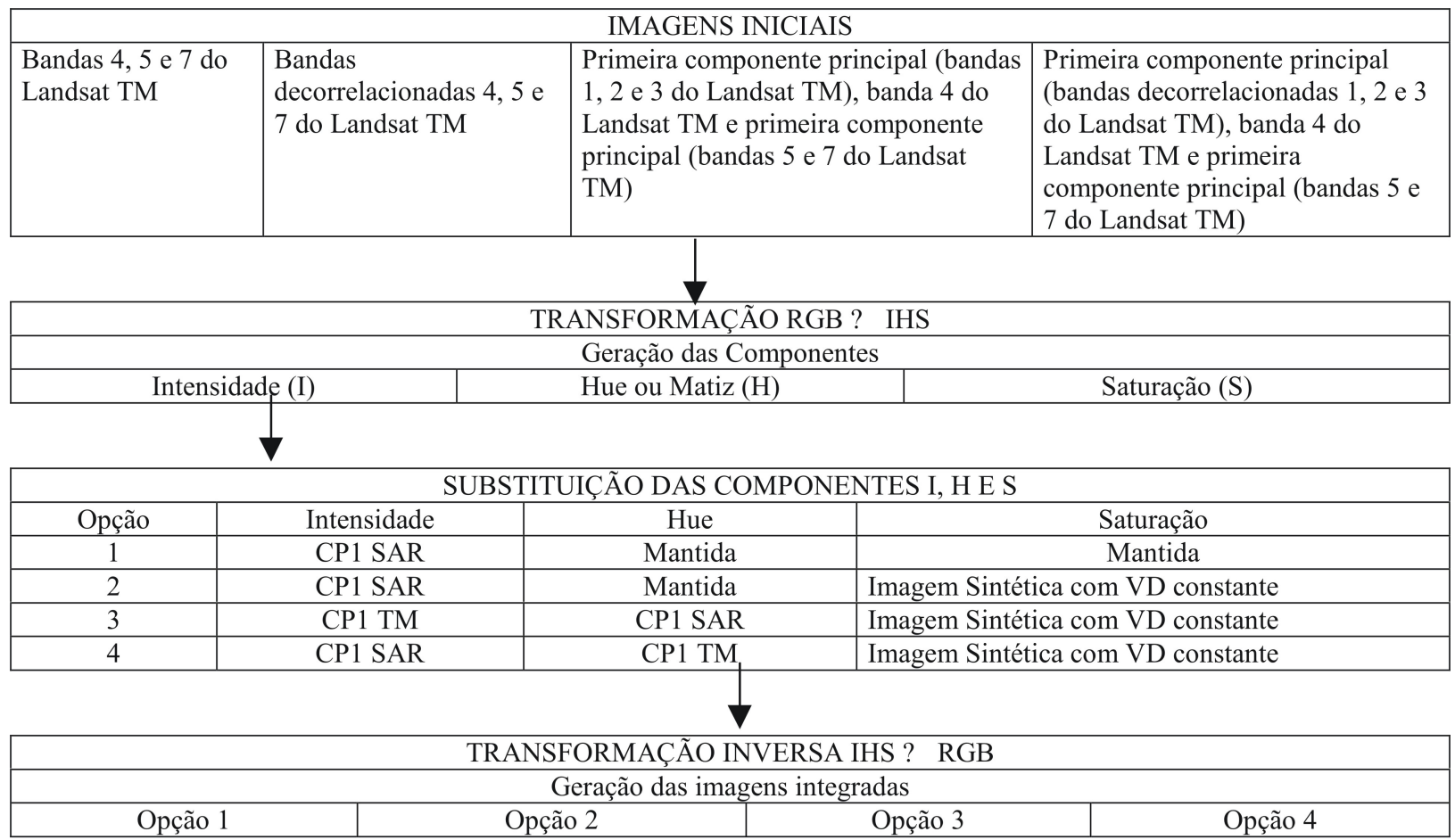

\section{Observações:}

- As imagens sintéticas possuem valores digitais (VD) constante. Foram testadas imagens com VD = 60, 80, 120 e 150.

- A primeira componente principal das imagens de SAR (CP1 SAR) foi gerada considerando-se todas as imagens de radar: RADARSAT, modos de imageamento S2 e S7, JERS-1 e GEMS-1.

A primeira componente principal do Landsat-TM (CP1 TM) foi gerada a partir da transformação por componentes principais das seis bandas do visível e infravermelho de ondas curtas do Landsat-TM (bandas 1, 2, 3, 4, 5 e 7).

pressiva para a discriminação de litotipos, além de favorecer a interpretação estrutural da área investigada. Por sua vez, as características espectrais (cromaticidade) dos dados ópticos auxiliaram mais na obtenção de informações litológicas associadas com uso do solo.

O método de integração utilizado permitiu a combinação de da- dos multi-sensores e minimizou os efeitos antrópicos, possibilitando o seu aproveitamento para mapeamento geológico. Isso pôde ser comprovado pelo índice de acurácia do mapa interpretado, que ficou em torno de 0.77 . A desvantagem desse método está no custo de aquisição das imagens de SAR, mais especificamente, do satélite RADARSAT. 


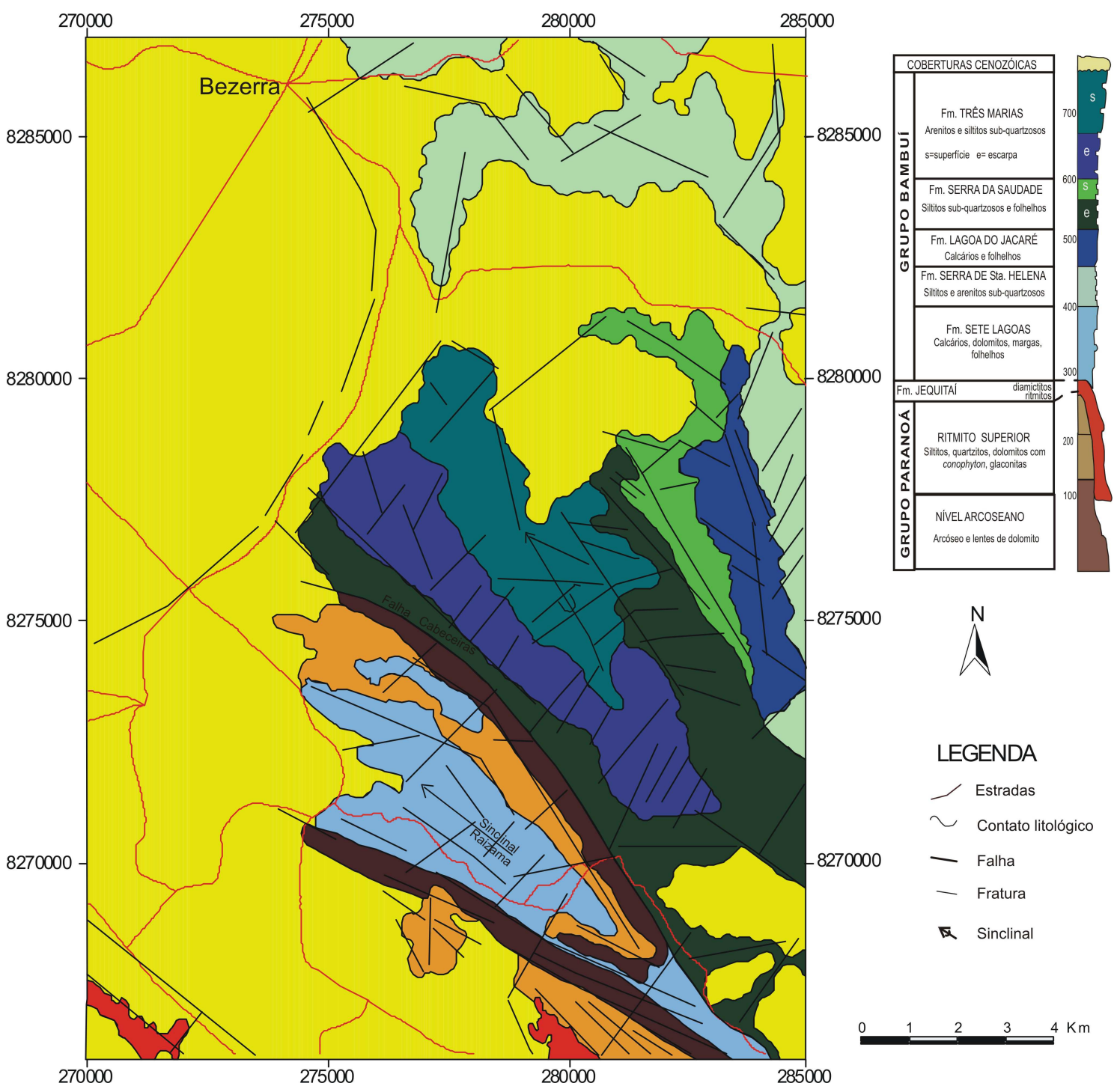

Figura 4 - Mapa geológico interpretado da área de estudo, baseado na imagem resultante da técnica de composição colorida por IHS ( I = CPI SAR; He S = DEC, bandas 4, 5 e 7 do Landsat 5-TM $)$.

Tabela 4 - Tabela de validação cruzada usando os mapas geológicos atualizado e interpretado da área de estudo. EC = Erro de Comissão; EO = Erro de Omissão; e AT = Acurácia Total.

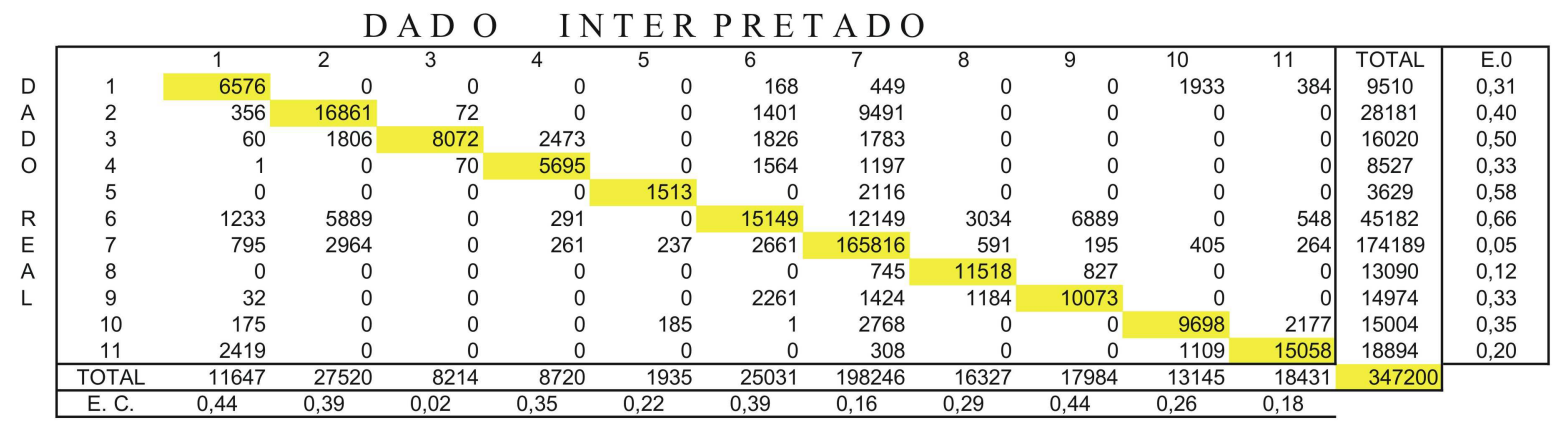

A. $\mathrm{T} .=0,77$

Unidades: 1 - Nível Arcoseano; 2 - Serra de Santa Helena; 3 - Lagoa do Jacaré; 4 - Serra da Saudade escarpa; 5 - Jequitaí; 6 Serra da Saudade superfície; 7 - Cobertura Cenozóica; 8 - Três Marias superior; 9 - Três Marias escarpa; 10 - Ritmito Superior; e 11 - Sete Lagoas. 
Agradecimentos À Threetek por ter cedido, em caráter provisório, o pacote de programas PCI, versão 6.3, e aos revisores e edito- res da RBG pelas sugestões ao manuscrito.

\section{Referências}

Amaral S. \& Shimabukuro Y.E. 1997. Sensoriamento Remoto por Radar (SAR): Pré-processamento de imagens RADARSAT ("Fine Mode") na região da Floresta Nacional do Tapajós. In: Latin-American Seminar Radar Remote Sensing - Image Processing Techniques, Buenos Aires, ESA, SP-407, Proceedings, pp. 37- 42.

Chaves J.M 2002. Discriminação de litotipos com base em dados de radar de abertura sintética e sinergismo radar/sistema óptico. Tese de Doutorado, Inst. Geociências. Universidade de Brasília. Brasília. $147 \mathrm{p}$.

Chavez Jr. P.S., Berlin G.L., Sowers L.B. 1982. Statistical method for selecting Landsat MSS ratios. J. App. Photogr. Engineering, 8:23-30.

Chavez Jr. P.S., Guptill S.C., Bowelli J.A. 1984. Image processing techniques for Thematic Mapper data. In: ASP Conference, Washington, DC. Proceedings... Washington, DC, American Society of Photogrammetry, pp. 728-752.

Chavez Jr. P.S. \& Kwarting A.Y. 1994. Extracting spectral contrast in Landsat Thematic Mapper image data using selective principal component analysis. Photogram. Enginee. Remote Sensing, 55:339-348.

Elachi C. 1988. Spaceborne radar remote sensing applications and techniques. New York: IEEE Press, 255 p.

Fraser S.J. 1991. Discrimination and identification of ferric oxides using satellite Thematic Mapper data: a Newman case study. Intern. J. Remote Sensing 12:635-641.

Frost V.S., Stiles J.A., Shanmugan K.S., Holtzman J.C. 1982. A model for radar images and its applications to adaptative digital filtering of multiplicative noise. IEEE Transac. Pattern Analysis Machine Intelligence, 4:157-166.

Gillespie A.R. 1980. Digital techniques of image enhancement. In: B.S Siegal \& A.R. Gillespie (eds). Remote Sensing in Geology. New York: John Wiley, Cap. 6, 139-226.

Gillespie A.B., Kahle A.B., Walker R.E. 1986. Colour enhancement of highly correlated images, I. Decorrelation and HIS contrast stretches. Remote Sensing of Environment 20:209-235.

Goward S.N., Masek J.G., Williams D.L., Irons J.R., Thompson R.J. 2001. The Landsat-7 mission: terrestrial research and applications for the 21 $1^{\text {st }}$ century. Remote Sensing of Environment 78:3-12.

Guimarães E. 1997. Estudos de proveniência e diagênese com ênfase na caracterização dos filossilicatos dos Grupos Paranoá e Bambui, na região de Bezerra-Cabeceiras (GO). Tese de Doutorado, Inst. de Geociências. Universidade de Brasília, 270 p.

Harris J.R. \& Murray R. 1990. IHS transform for the integration of radar imagery with other remotely sensed data. Photogr. Enginee. Remote Sensing, 56:1631-1641.

Henderson F.M. \& Lewis A.J. 1998. Principles and Applications of Imaging Radar. Manual of Remote Sensing, Manual of Remote Sensing. ASPRS, New York, Jonh Wiley \& Sons, $3^{\mathrm{a}}$ ed, Vol. 2, 656 p.

Kaufman Y.J. \& Sendra C. 1988. Algorithm for automatic atmospheric corrections to visible and near-IR satellite imagery. Intern. J. Remote Sensing 9:1357-1381.

Krejci L.C., Fortunato F.F., Corrëa P.R.S. 1982. Pedologia: Levantamento Exploratório de Solos. Folha Brasília, Projeto RADAMBRASIL, 29: 297-460.

Lauer D.T., Morain S.A., Salomonson V.V. 1997. The Landsat program: its origins, evolution, and impacts. Photogram. Engine. Remote Sensing. 63:831-838.
Lewis A.J., Henderson F.M., Holcomb D.W. 1998. Radar fundamentals: the geoscience perspective. In: F.M. Henderson \& A.J. Lewis (ed.) Principles \& Applications of Imaging Radar. Manual of Remote Sensing. Manual of Remote Sensing, ASPRS, New York, Jonh Wiley \& Sons, $3^{\text {a }}$ ed, Vol. 2, 131-180.

Meneses P.R. \& Ferreira Jr. L.G. 2001. Comportamento espectral de minerais e rochas. In: P.R. Meneses \& J.S. Madeira Netto (org.) Sensoriamento Remoto: Reflectância dos Alvos Naturais. UnB, Brasília; Embrapa Cerrados: Planaltina, Cap. 3, 63-123.

Meneses P.R, Assad E.D., Sano E.E. 1995. Introdução ao Processamento de Imagens de Satélites de Sensoriamento Remoto. Textos Universitários. Brasília: UnB, 96 p.

Mika A.M. 1997. Three decades of Landsat instruments. Photogram. Engine. Remote Sensing, 63:839-852.

Moran M.S., Hymer D.C., Qi J., Kerr Y. 2002. Comparison of ERS-2 SAR and Landsat 5-TM imagery for monitoring agricultural crop and soil conditions. Remote Sensing of Environment 79:243-252.

Paradella W.R., Santos A.R., Dall'Agnol R., Pietsch R.W., Sant'Anna M.V. 1998. A geological investigation based on airborne (SAREX) and spaceborne (RADARSAT) SAR integrated products in the central Serra dos Carajás granite area, Brazil. Can. J. Remote Sensing 21:376-392.

Paradella W.R., Santos A.R., Veneziani P., Morais M.C. 2000. Synthetic aperture radar for geological applications in the moist tropics: experiences from the Brazilian Amazon region. Rev. Bras. Geoc., 30:534-538.

Paradella W.R., Santos A.R., Veneziani P., Morais M.C. 2001. O sinergismo de imagens standard descendente do RADARSAT-1 e Thematic Mapper do LANDSAT-5 em aplicação geológica na Amazônia. In: Simp. Bras. Sensor. Remoto, 10, Foz do Iguaçu, PR. Anais..., Inpe. (versão em CD-ROM).

PCI. 1999. EASI-PACE User'S Manual. versión 6.3, Ontario, PCI Enterprises. 362 p.

Pietsch R.W. 1993. TFI SAR processing report. Internal Report, CCRS/ Dendron Surveying, Ottawa, Canada.

Raney K. 1998. Radar Fundamentals: Technical Perspective. In: Henderson, F.M., Lewis, A.J. 1998. Principles and Applications of Imaging Radar. Manual of Remote Sensing. ASPRS, New York, Jonh Wiley \& Sons, Vol. 2, 3ª ed., 9-130.

Silva A.B. 1999. Sistemas de Informações Georreferenciadas: Conceitos e Fundamentos. Campinas, Unicamp, $250 \mathrm{p}$.

Singhroy V.H. 1992. Radar geology: techniques and results. Episodies, 15:15-20.

Souza Filho C.R. \& Drury S.A. 1998. Evaluation of JERS-1 (FUYO-1) OPS and Landsat TM images for mapping of gneissic rocks in arid areas. Intern. J. Remote Sensing, 19:3569-3594.

Teillet P.M. \& Fedosejevs G. 1995. On the dark target approach to atmospheric correction of remotely sensed data. Can. J. Remote Sensing, 21:374-387.

Werle D. 1998. Radar Remote Sensing: A Trainnig Manual. Dendron Resource Surveys Inc., Ottawa, Canada, 213 p.

Manuscrito SR-18

Recebido em 01 de novembro de 2002 Revisão dos autores em 06 de matço de 2003 Revisão aceita em 20 de abril de 2003 